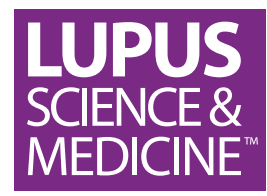

\title{
Remission and low disease activity in systemic lupus erythematosus: an achievable goal even with fewer steroids? Real-life data from a monocentric cohort
}

\author{
Chiara Tani, Roberta Vagelli, Chiara Stagnaro, Linda Carli, Marta Mosca
}

To cite: Tani $C$, Vagelli $R$, Stagnaro C, et al. Remission and low disease activity in systemic lupus erythematosus: an achievable goal even with fewer steroids? Real-life data from a monocentric cohort. Lupus Science \& Medicine 2018;5:e000234. doi:10.1136/ lupus-2017-000234

Received 24 July 2017 Revised 16 0ctober 2017 Accepted 3 November 2017

Check for updates

Rheumatology Unit, Department of Clinical and Experimental Medicine, University of Pisa Pisa, Italy

Correspondence to

Dr Marta Mosca; marta.mosca@ med.unipi.it

\begin{abstract}
Objectives To evaluate what proportion of patients fulfil the DORIS definition of remission, the definition of lupus low disease activity state (LLDAS) and LLDAS with a glucocorticoid (GC) dosage $\leq 5$ (LLDAS5) in a longitudinal monocentric cohort of patients with SLE; to identify predictors of sustained remission and LLDAS attainment; to evaluate the effect of sustained remission and LLDAS on damage accrual over a period of 5 years and compare the two conditions in terms of clinical outcomes.

Methods Retrospective analysis of data prospectively collected from patients with SLE followed from 2012 to 2016.

Results 115 patients were included in this analysis. At baseline, $72 \%$ of patients were on LLDAS and almost all patients also fulfilled the LLDAS5 definition; $45 \%$ of patients were in remission on treatment, $12 \%$ were in remission off treatment, $26 \%$ were in complete remission on treatment, $2 \%$ were in complete remission off treatment. Disease activity at baseline was the strongest predictor of subsequent LLDAS and remission; the presence of joint and cutaneous manifestations was associated with a minor likelihood to achieve LLDAS or remission during follow-up. Patients in remission and LLDAS for the whole follow-up period accrued significantly less organ damage; on the contrary, patients who maintained all kinds of remissions or LLDAS for less than $50 \%$ of the time did not show any differences in damage accrual with respect to the rest of the cohort.

Conclusion Remission and LLDAS, even with reduced GC use, are an achievable goal in clinical practice; sustained LLDAS and remission are both associated with reduced damage accrual.
\end{abstract}

\section{INTRODUCTION}

SLE is a chronic disease with a relapsing remitting course. Disease activity over time is associated with damage accrual, poor longterm outcomes and comorbidities. ${ }^{1-3}$ Thus, the control of disease activity is an important target in the treatment of patients with SLE. ${ }^{4}$

In 2016, an international expert panel (the Definition of Remission in SLE, DORIS project) agreed on three definitions of remission in SLE: complete remission (with negative serology), clinical remission on therapy (clinical ROT) and complete remission on therapy (complete ROT).$^{5}$

More recently, low disease activity (LDA) has also been proposed as an alternative goal in the treat-to-target strategy and different definitions of this state have been proposed and applied to different SLE cohorts. ${ }^{6-8}$

In 2015, the lupus low disease activity state (LLDAS) was developed as a consensus-based definition of minimally acceptable disease activity in patients with SLE. ${ }^{7}$ This definition was initially tested for criterion validity in a first multiethnic cohort of patients showing that it is associated with improved patient outcomes. $^{7910}$

The LLDAS set up the maximum glucocorticoid (GC) dosage admitted at $\leq 7.5 \mathrm{mg}$ of prednisone daily. The risk of irreversible organ damage increases as the prednisone dose increases and $\leq 7.5$ prednisone/day is traditionally considered a low dosage and an 'acceptable' dose to be used long term. On the other hand, however, some data have shown that compared with no prednisone use, the increase in risk is small or inexistent only for a cumulative average dose below $180 \mathrm{mg} / \mathrm{month}$, that is to say, $6 \mathrm{mg} /$ day. ${ }^{11}$ Other studies demonstrated that long-term prednisone doses $\geq 5 \mathrm{mg}$ are associated with a significant risk in bone loss and fractures, arterial hypertension, myocardial infarction and serious infections. ${ }^{12-15}$ Furthermore, it has been suggested that hyperglycaemia may appear at doses as low as $2.5 \mathrm{mg} /$ day of prednisone equivalent. ${ }^{16}$

Thus, a 'safe' dose of GC is still to be defined and every effort should be made to keep GC at the lowest dosage and, if possible, to withdraw it. ${ }^{4}$ 
The aims of this study are: (1) to evaluate what proportion of patients fulfil the DORIS definition of remission and the definition of LLDAS in a longitudinal monocentric cohort of patients with SLE; (2) to evaluate what proportion of patients fulfil the definition of LLDAS with a GC dosage $\leq 5$ (LLDAS5); (3) to identify the predictors of sustained remission and LDA; and (4) to evaluate the effect of sustained remission and LDA on damage accrual over a period of 5 years and compare the two conditions in terms of clinical outcomes.

\section{METHODS \\ Patients}

The Pisa lupus clinic cohort is a longitudinal observational cohort of patients with SLE set up in January 2012; clinical and serological variables are collected from patients who are regularly followed at the clinic. The cohort includes adult patients with SLE satisfying the 1997 American College of Rheumatology classification criteria. Both outpatient visits and hospital admissions for disease flares or disease complications are included in the database.

This is a retrospective analysis of data prospectively collected from patients with SLE with at least one visit per year and complete clinical and serological data followed between 2012 and 2016. When patients were assessed more than once per year, the evaluation with higher disease activity was considered.

\section{Case definitions and outcome measures}

Disease activity was recorded according to validated activity indices (Safety of Estrogens in Lupus Erythematosus: National Assessment-SLE Disease Activity Index (SLEDAI) and European Consensus Lupus Activity Measurements (ECLAM)) as well as physician's judgement on a 0-3 scale (PhGA).

The definitions of remission were applied according to the DORIS definitions.

To simplify the analysis, the definitions were also grouped into the following categories:

A. clinical remission ON treatment (RONT),

B. clinical remission OFF treatment (ROFT),

C. clinical remission with negative serology ON treatment (complete RONT),

D. clinical remission with negative serology OFF treatment (complete ROFT).

The definitions of LLDAS and LLDAS5 were applied to identify patients with LDA.

Sustained remission and sustained LDA were defined when patients satisfied the definition at all assessments of the study period.

Organ damage was calculated using the Systemic Lupus International Collaborating Clinics Damage Index (SLICC-DI) score (SDI) at study entry and at the last observation.

\section{Statistical analysis}

Continuous variables are reported as means and SDs or median and 25-75 IQR for non-normally distributed data.
Differences between groups were calculated by Student's t-test or Mann-Witney U test as appropriate.

Categorical variables are reported as proportions and are compared using $\mathrm{X}^{2}$ for binary outcomes. Multivariate analysis was also performed including all covariates that were significantly associated with the outcomes at univariate analysis. Only subsequent evaluations were considered for the analysis of predictors when the baseline predictive variable was also included in the outcome measure (ie, SLEDAI score).

Baseline variables considered in the univariate analysis of predictors were the following: age, disease duration, SLEDAI score, serological activity, previous organ involvement, ongoing manifestations (articular manifestations, mucocutaneous manifestations, renal manifestations, neuropsychiatric manifestations, serositis, haematological manifestations), therapy with hydroxychloroquine.

To evaluate the degree of agreement between the different definitions used for remission based on the SLEDAI score, ECLAM or clinical judgement was evaluated estimating the Cohen's kappa; this analysis was performed for all the baseline assessments. K values $>0.80$ were considered to be a very good level of agreement.

$P$ value $<0.05$ is considered statistically significant. All the analyses were performed using STATA V.13.

\section{RESULTS}

\section{Baseline evaluations}

The Pisa SLE cohort includes 310 patients regularly followed; among these, 115 patients were eligible for the study and were included in this analysis. All included patients had a follow-up of at least 5 years (2012-2016). One hundred and six (93\%) were female, mean age and disease duration at baseline visit were $41.6 \pm 13$ years and $12.9 \pm 9$ years, respectively. Twelve patients $(10.3 \%)$ had a disease duration of less than 1 year at enrolment. Sixty-one patients $(53.5 \%)$ had a history of major organ involvement (renal and/or neuropsychiatric manifestations).

The median number of visits/year was 2.5 (minimum 1-maximum 4, IQR 2-3); a minority of patients attended only one visit $(15 \%)$.

Baseline clinical data, disease activity and ongoing therapies are reported in table 1 . Of note, articular manifestations, serositis and haematological involvement were the most common ongoing manifestations $(20.3 \%$ and $17 \%$, respectively). Active major organ involvement was present in only a minority of patients (kidney involvement in eight patients and neuropsychiatric involvement in one patient); on the other hand, 31\% presented only serological activity (anti-dsDNA and/or hypocomplementaemia).

The majority of patients (84\%) were on GC therapy (mean dosage $6.25 \pm 4.1 \mathrm{mg} /$ day prednisone equivalent) and $69.5 \%$ were taking a daily prednisone dosage $\leq 5 \mathrm{mg}$. $71.8 \%$ were taking antimalarials (mean dosage $225 \pm 70 \mathrm{mg}$ /day), $43.4 \%$ were receiving immunosuppressive drugs while only a minority $(0.8 \%)$ were on biologics. 
Table 1 Ongoing clinical manifestations, disease activity assessments and therapies at baseline in the whole cohort

Whole cohort

\begin{tabular}{ll}
\hline Renal manifestations (\%) & 8 \\
\hline Articular manifestations (\%) & 20.3 \\
\hline Mucocutaneous manifestations (\%) & 13.4 \\
Neuropsychiatric manifestations (\%) & 0.8 \\
Serositis/haematological manifestations (\%) & 17 \\
SLEDAI (median, IQR) & $2(0-4)$ \\
SLEDAl=0 (\%) & 31.3 \\
SLEDAI>4 (\%) & 24.3 \\
Serological activity (\%) & 31 \\
Glucocorticoids (\%) & 84 \\
Immunosuppressants (\%) & 43.4 \\
Biologics (\%) & 0.8 \\
Antimalarials (\%) & 71.7 \\
\hline
\end{tabular}

SLEDAI, SLE Disease Activity Index.

The percentages of patients fulfilling LLDAS, LLDAS5 and DORIS definitions are reported in table 2.

At baseline visit, $72 \%$ of patients were on LLDAS; in addition, almost all patients fulfilling the LLDAS definition also fulfilled the LLDAS5 definition.

Irrespective of the definition used for disease activity, $>45 \%$ of patients were in remission on treatment, $12 \%$ were in remission off treatment, $26 \%$ were in complete remission on treatment and $2 \%$ were in complete remission off treatment. Interestingly, a very good agreement was found between the DORIS definitions using the ECLAM score, the SLEDAI score and the clinical judgement, especially for remission and complete remission off treatment $(\mathrm{k}>0.89, \mathrm{P}<0.001$ in all the tests performed).

\section{Follow-up}

At last observation, the median SLEDAI score was 2 (IQR 2-4); 32 patients (27.8\%) had an SLEDAI $=0$ and
$21(18.26 \%)$ had an SLEDAI score $>4$. Seventy-six patients $(66 \%)$ were still on GC treatment (mean dosage $6 \pm 3.2 \mathrm{mg} /$ day prednisone equivalent), $45(39 \%)$ were on IS, $10(8.7 \%)$ were on biologics and $95(82.6 \%)$ were on antimalarials. Twenty-six patients $(22.6 \%)$ were off treatment or were taking only antimalarials.

The percentages of patients fulfilling the definitions of remission at each annual evaluation are reported in table 2. Clinical remission on treatment is observed in about $45 \%$ of patients every year during the follow-up period, while complete remission on treatment was reported in 19\%-28\% of patients. In $10 \%-20 \%$ of patients the definition of remission off treatment was satisfied while complete remission off treatment was rare being observed in a small percentage of patients ranging from $1 \%$ to $7 \%$ during the follow-up period.

Over the 5 years of follow-up, sustained remission on treatment was recorded in $21 \%$ of patients and complete remission on treatment was rare $(7.8 \%$ of patients). Remission off treatment was rare; in detail a sustained remission off treatment was observed in $5.2 \%$ of patients and complete remission off treatment in $0.8 \%$.

As far as LDA is concerned, both LLDAS and LLDAS5 were attained in more than $70 \%$ of the cohort right through to follow-up; however, only $36.5 \%$ and $35.3 \%$ of the patients maintained the state for the whole duration of the observation.

\section{Predictors of sustained remission and sustained LLDAS}

Baseline variables that are associated with sustained remission and LLDAS are reported in table 3.

Patients who maintained remission on treatment during the follow-up had lower disease activity scores at baseline $(\mathrm{P}<0.001)$ and were not presenting joint $(\mathrm{P}=0.01)$ or mucocutaneous involvement $(\mathrm{P}=0.02)$. Patients who achieved a sustained complete remission on treatment presented lower disease activity scores at baseline $(\mathrm{P}=0.02)$ and they were more likely to have serological activity without clinical manifestations $(\mathrm{P}=0.04)$. Similarly, remission off treatment was correlated with serologically active but clinically quiescent disease at

Table 2 Patients fulfilling LLDAS, LLDAS5 and DORIS criteria at baseline evaluation and during follow-up

\begin{tabular}{llllllll} 
& $\begin{array}{l}\text { Baseline } \\
\mathbf{2 0 1 2}\end{array}$ & $\mathbf{2 0 1 3}$ & $\mathbf{2 0 1 4}$ & $\mathbf{2 0 1 5}$ & $\begin{array}{l}\text { Last visit } \\
\mathbf{2 0 1 6}\end{array}$ & $\begin{array}{l}\text { Sustained } \\
\mathbf{1 0 0 \%}\end{array}$ & $\begin{array}{l}\text { Sustained } \\
\mathbf{5 0 \%}\end{array}$ \\
\hline LLDAS & $72(63.1 \%)$ & $72(63.1 \%)$ & $80(70.2 \%)$ & $77(68.1 \%)$ & $86(74.8 \%)$ & $42(36.5 \%)$ & $81(70.4 \%)$ \\
LLDAS5 & $71(61.6 \%)$ & $69(60 \%)$ & $80(70.2 \%)$ & $77(68.1 \%)$ & $85(73.9 \%)$ & $41(35.6 \%)$ & $79(68.6 \%)$ \\
RONT & $57(49.6 \%)$ & $53(46 \%)$ & $60(52.1 \%)$ & $55(47.8 \%)$ & $55(47.8 \%)$ & $25(21.7 \%)$ & $55(47.8 \%)$ \\
ROFT & $12(10.4 \%)$ & $14(12.1 \%)$ & $18(15.6 \%)$ & $23(20 \%)$ & $23(20 \%)$ & $6(5.2 \%)$ & $17(14.8 \%)$ \\
$\begin{array}{l}\text { Complete } \\
\text { RONT }\end{array}$ & $33(28.7 \%)$ & $30(26 \%)$ & $31(26.9 \%)$ & $22(19.1 \%)$ & $24(20.8 \%)$ & $9(7.8 \%)$ & $24(20.9 \%)$ \\
Complete & $2(1.7 \%)$ & $4(3.5 \%)$ & $8(6.9 \%)$ & $8(6.9 \%)$ & $9(7.8 \%)$ & $1(0.8 \%)$ & $4(3.5 \%)$ \\
ROFT & & & & & & &
\end{tabular}

DORIS, Definition of Remission in SLE; LLDAS, lupus low disease activity state; LLDAS5, LLDAS with a GC dosage $\leq 5$; ROFT, remission OFF treatment; RONT, remission ON treatment. 
Table 3 Univariate analysis showing baseline predictors of sustained LLDAS, LLDAS5 and remission according to DORIS definitions

\begin{tabular}{lllllll}
\hline Baseline predictors & LLDAS & LDAS5 & RONT & ROFT & $\begin{array}{l}\text { Complete } \\
\text { RONT }\end{array}$ & $\begin{array}{l}\text { Complete } \\
\text { ROFT }\end{array}$ \\
\hline Age & NS & NS & NS & NS & NS & NS \\
Disease duration & NS & NS & NS & NS & NS & NS \\
Recent onset & NS & NS & NS & NS & NS & NS \\
Mean SLEDAl & $\mathrm{P}<0.001$ & $\mathrm{P}<0.001$ & $\mathrm{P}=0.001$ & $\mathrm{NS}$ & $\mathrm{P}=0.02$ & $\mathrm{NS}$ \\
SLEDAl>4 & $\mathrm{P}<0.001$ & $\mathrm{P}<0.001$ & $\mathrm{P}=0.001$ & $\mathrm{NS}$ & $\mathrm{P}=0.07$ & $\mathrm{NS}$ \\
Only serological activity & $\mathrm{NS}$ & $\mathrm{NS}$ & $\mathrm{NS}$ & $\mathrm{P}=0.015$ & $\mathrm{P}=0.04$ & $\mathrm{NS}$ \\
Previous major organ involvement & $\mathrm{NS}$ & $\mathrm{NS}$ & & $\mathrm{NS}$ & $\mathrm{NS}$ & $\mathrm{NS}$ \\
Articular manifestations & $\mathrm{P}=0.004$ & $\mathrm{P}<0.001$ & $\mathrm{P}=0.01$ & $\mathrm{NS}$ & $\mathrm{NS}$ & $\mathrm{NS}$ \\
Mucocutaneous manifestations & $\mathrm{P}=0.02$ & $\mathrm{P}=0.12$ & $\mathrm{P}=0.02$ & $\mathrm{NS}$ & $\mathrm{NS}$ & $\mathrm{NS}$ \\
Renal manifestations & $\mathrm{NS}$ & $\mathrm{P}=0.02$ & $\mathrm{NS}$ & $\mathrm{NS}$ & $\mathrm{NS}$ & $\mathrm{NS}$ \\
Neuropsychiatric manifestations & $\mathrm{NS}$ & $\mathrm{NS}$ & $\mathrm{NS}$ & $\mathrm{NS}$ & $\mathrm{NS}$ & $\mathrm{NS}$ \\
Serositis/haematological manifestations & $\mathrm{NS}$ & $\mathrm{NS}$ & $\mathrm{NS}$ & $\mathrm{NS}$ & $\mathrm{NS}$ & $\mathrm{NS}$ \\
Therapy with HCQ & $\mathrm{NS}$ & $\mathrm{NS}$ & $\mathrm{NS}$ & $\mathrm{NS}$ & $\mathrm{NS}$ & $\mathrm{NS}$ \\
\hline
\end{tabular}

DORIS, Definition of Remission in SLE; HCQ, hydroxychloroquine; LLDAS, lupus low disease activity state; LLDAS5, LLDAS with a GC dosage $\leq 5$; NS, not significant; ROFT, remission OFF treatment; RONT, remission ON treatment; SLEDAI, SLE Disease Activity Index.

baseline $(\mathrm{P}=0.015)$. With the limitation of the small number of patients who maintained complete remission off treatment, no statistically significant associations were found between baseline variables and sustained complete remission of treatment attainment.

Sustained LLDAS during the follow-up was associated with a lower SLEDAI score at study entry $(\mathrm{P}<0.001)$ and patients were not complaining active joint symptoms $(\mathrm{P}=0.004)$ nor skin manifestations $(\mathrm{P}=0.02)$. They also tended to have fewer renal manifestations at study entry $(\mathrm{P}=0.08)$. Similarly, patients who maintained LLDAS5 were more likely to be inactive at baseline with a significantly lower SLEDAI $(\mathrm{P}<0.001)$ and did not complain of joint $(\mathrm{P}<0.001)$ or cutaneous symptoms $(\mathrm{P}=0.012)$ and, in this case, they had significantly less frequent renal involvement at study entry $(\mathrm{P}=0.02)$.

No other significant predictors of LLDAS or remission were found among the epidemiological and clinical variables tested.

\section{Damage accrual}

At study entry, 49 patients (42\%) had at least one item of the SDI score; among these the median SDI score was 2 (IQR 1-3). Overall, a total of 99 SLICC items was recorded and 29 (29\%) can be considered GC-related damage (cataract, osteoporosis with fractures, muscle atrophy or weakness, avascular necrosis).

At the last observation, $59(50.8 \%)$ patients had at least one organ damage; 37 patients $(31.9 \%)$ accrued organ damage during the follow-up; in those patients, the median increase in SDI was 1 (IQR 1-2) resulting in a median final SDI score of 2 (IQR 1-4). At the last observation, a total of 151 items of damage was recorded and 50 of these (33\%) were related to chronic GC usage.

Patients in clinical remission on treatment for the whole follow-up period accrued significantly less organ damage ( $\triangle$ SLICC 0.12 vs $0.48, \mathrm{P}=0.018$ ); however, patients who maintained clinical remission on treatment for less than $50 \%$ of the time did not show any differences in damage accrual with respect to the rest of the cohort.

The small number of patients who maintained remission off treatment or complete remission (both on and off treatment) impeded the analysis for these groups.

Patients who maintained LLDAS for the whole follow-up period accrued significantly less organ damage with respect to the rest of the cohort (mean $\triangle$ SLICC 0.11 vs $0.63, \mathrm{P}<0.001$ ) and they were more likely off treatment at the last observation $(\mathrm{P}=0.03)$. However, patients who maintained LLDAS for less than $50 \%$ of the time $(<2.5$ years $)$ accrued more organ damage (mean $\triangle$ SLICC $0.78 \pm 0.14$ vs $0.25 \pm 0.64, \mathrm{P}=0.0004$ ) with respect to the patients who maintained LLDAS for more than 2.5 years.

No differences in damage accrual were observed between sustained LLDAS and LLDAS5 (mean $\triangle$ SLICC 0.11 vs $0.12, \mathrm{P}=\mathrm{NS}$ ).

In the multivariate analysis, by adjusting for age, disease duration, baseline SLICC and the presence of previous severe organ involvement (renal and/or neuropsychiatric) for both sustained remission on treatment and sustained LLDAS, the association with lower damage accrual was maintained $(\mathrm{P}=0.02$ and $\mathrm{P}=0.002$, respectively).

The complete list of the SLICC items recorded at baseline evaluation and at the end of follow-up is reported in 


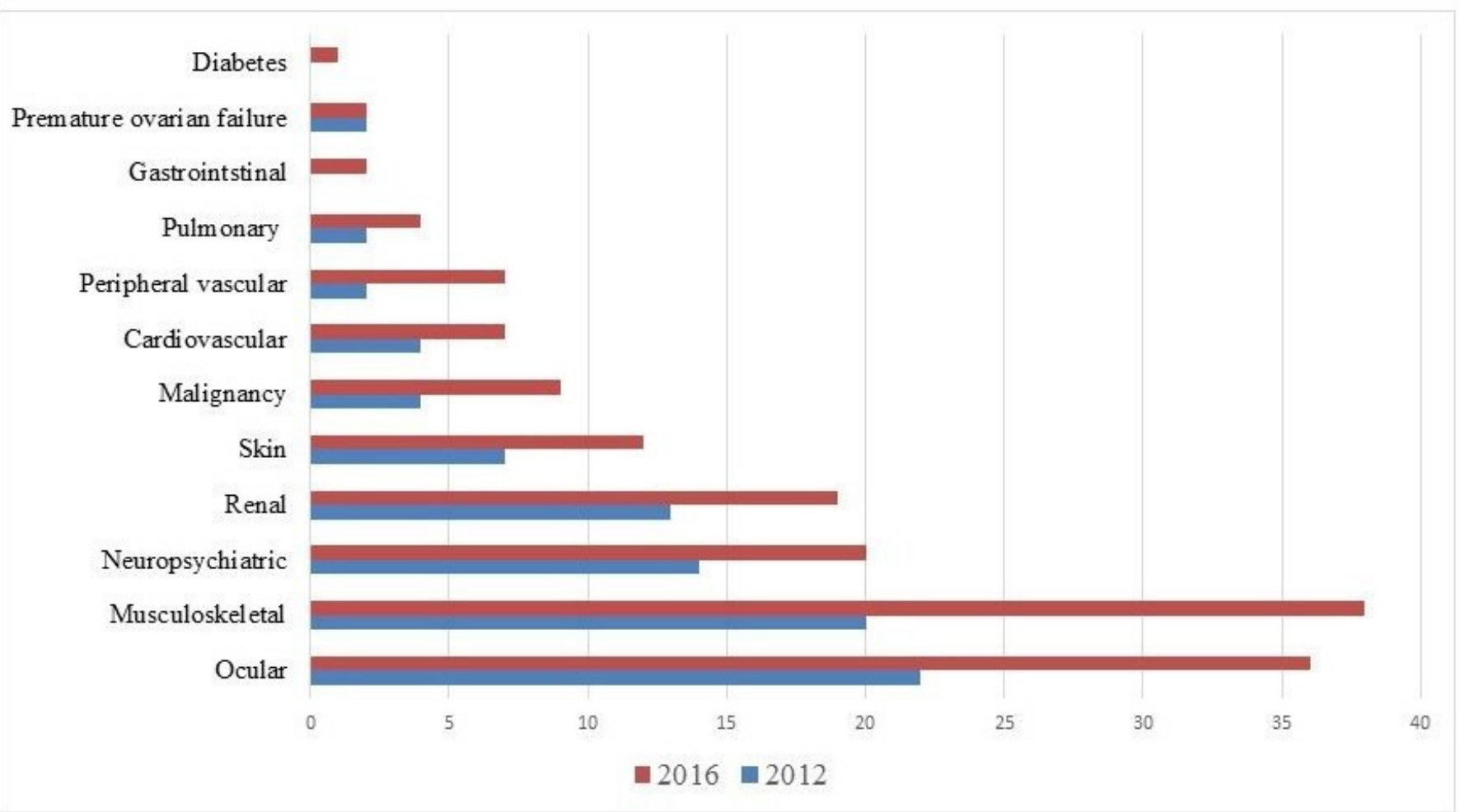

Figure 1 Items of the Systemic Lupus International Collaborating Clinics Damage Index (SLICC-DI) at baseline and at the last observation.

figure 1. Of note, 31 items were GC related at study entry, 50 at last observation.

\section{DISCUSSION}

Disease remission is considered the ideal target for treatment in patients with SLE in clinical trials and in clinical practice; more recently, the idea is emerging that an LDA state might be considered a good alternative target. ${ }^{4}$ Several definitions for remissions and LDA have been proposed, generating a great scientific debate. ${ }^{68917-23}$

In this study, we applied the DORIS definitions of remission and the LLDAS definition to our cohort of patients in order to define their frequency in our clinical practice.

At the first evaluation, half of patients were in remission on treatment and about $25 \%$ were in clinical and serological remissions. Clinical remission off treatment was uncommon $(10 \%-13 \%)$ and complete remission off treatment was rare $(<2 \%)$; clinical remission off treatment was less common $(10 \%-13 \%)$ and complete remission off treatment $(<2 \%)$ was very rare. However, if we consider the entire 5 years of follow-up, only $22 \%$ of patients maintained the remission state on treatment. Durable remissions off treatment have rarely been observed $(5.2 \%$ and $<1 \%$ for clinical remission and complete remission, respectively). In summary, our data suggest that in clinical practice remission is an achievable goal, but its maintenance over time can be difficult.

These results are in line with previous reports showing a very low frequency of sustained remission off treatment in different SLE cohorts. ${ }^{17-24}$ Of note, as would be expected, when progressively less stringent criteria were applied to the remission definition, remission frequency increased. ${ }^{20} 22$

The percentage of patients fulfilling the criteria for LLDAS is high at baseline and at each annual evaluation (63\%-74.8\%). Interestingly, almost all patients fulfilling the LLDAS definition also fulfilled the LLDAS5 definition in our cohort; thus, trying to keep the lower GC dose of $5 \mathrm{mg}$ /day is also an achievable goal in clinical practice.

This result is similar to what was recently registered in a large multiethnic cohort of patients from Asian-Pacific countries at a single point in time $(44 \%){ }^{10}$

However, sustained LLDAS lasting for the whole 5-year follow-up was less frequent in our cohort $(36.5 \%)$ while $70 \%$ of our patients maintained the LLDAS for less than $50 \%$ of the time. The same results were registered when considering LLDAS5.

In a retrospective analysis of 183 patients followed for a median time of 5 years, Tsang-A-Sjoe et al reported a frequency of LLDAS lasting for $\geq 50 \%$ of follow-up in $64.5 \%$ of patients. ${ }^{25}$

More recently, Polachek et al applied a definition of LDA based on the SLEDAI-2K disease activity score and they found that $43.7 \%$ of patients from their prospective cohort fulfilled this definition for at least 1 year. ${ }^{9}$

In our cohort, disease activity at baseline proved to be the strongest predictors of subsequent LLDAS and remission; on the contrary, the presence of joint and cutaneous manifestations at baseline was associated with a minor likelihood of obtaining LDA or remission during 
the following years. If we consider only the patients who attained an LLDAS5, they also are less likely to present renal involvement at baseline. This aspect could signify that the presence of renal involvement still represents a major requirement for higher GC doses.

These results might suggest that the control of some disease manifestations (ie, joint and skin symptoms) is still suboptimal or a physician's compromise in 'accepting' some degree of activity in non-life-threatening organs such as joints and skin.

Several studies demonstrated that disease remission is associated with better short-term and long-term outcomes such as organ damage accrual, thus disease remission is considered the clinical target to be pursued. ${ }^{42126}$ In our cohort, patients who maintained clinical remission (both on treatment and off treatment), irrespective of serology, accrued less organ damage during follow-up; interestingly, if remission was maintained for less than $50 \%$ of the period this advantage was lost, thus suggesting that the duration of the remission is a crucial point to be considered. This has also been recently demonstrated by Zen et al who showed that $\geq 2$ consecutive year remission was protective against damage accrual. ${ }^{27}$

Sustained LLDAS was also associated with better 5-year outcomes in terms of damage accrual. This result confirms what has already been observed in the large retrospective Asian-Pacific cohort that validated the LLDAS criteria. Even considering the difference in LDA used, these data are also in line with the work by Polachek et al who reported similar short-term outcomes in the subgroups of patients with LDA and remission with respect to patients with high disease activity. If confirmed in future longterm studies, these data suggest that LDA might be used as a reliable outcome measure in therapeutic trials or in treat-to-target strategies.

In our opinion, other interesting points have been raised by this analysis.

First, we observed a high agreement between the results obtained by applying the remission definition based on the two disease activity scores SLEDAI and ECLAM, thus suggesting that, in clinical practice, the physician might have the possibility to choose the most familiar disease activity score without losing information but improving the scoring accuracy and the feasibility of the criteria.

Second, our data highlight the fact that a GC dose 'less than $5 \mathrm{mg}$ /day' could be feasibly attempted in our patients; further studies are necessary to confirm that it will provide better clinical outcomes.

Some limitations of the study should be also acknowledged.

First of all, our sample is mainly composed of patients with long-standing disease; we recognise that this aspect might have a significant impact on the generalisability of the results to other cohorts with different characteristics. Further studies on newly diagnosed patients with SLE are ongoing.

Second, a minority of patients attended only one visit $(15 \%)$ per year. In our opinion, it is very unlikely that a disease flare is not captured by our evaluations; on the other hand, it is possible that patients in remission will attend fewer visits and, probably, the subgroup of patients with only one evaluation is a subgroup of patients with lower disease activity.

In conclusion, remission and LDA are achievable targets in clinical practice, although more stringent definitions of remission are achieved less frequently and rarely maintained during follow-up. Persistence of remission and LLDAS/LLDAS5 is associated with reduced damage accrual.

Thus, it can be hypothesised that remission or LDA needs to be a durable state to be considered a desirable treatment outcome; in this scenario, a tight control of the disease activity and a treat-to-target approach are essential supportive strategies.

Acknowledgements We thank Dr Wendy Doherty for her English language revision of the manuscript.

Contributors CT and MM substantially contributed to the conception of the work. CS, LC and RV participated in the acquisition, analysis and interpretation of data. CT and RV drafted the work. MM critically revised the paper. All authors approved the version of the manuscript submitted.

Competing interests None declared.

Ethics approval In accordance with the policy of our institution, ethical approval from our Research Ethics Board for this type of study was not required.

Provenance and peer review Not commissioned; externally peer reviewed. Data sharing statement There are no additional unpublished data from the study.

Open Access This is an Open Access article distributed in accordance with the Creative Commons Attribution Non Commercial (CC BY-NC 4.0) license, which permits others to distribute, remix, adapt, build upon this work non-commercially, and license their derivative works on different terms, provided the original work is properly cited and the use is non-commercial. See: http://creativecommons.org/ licenses/by-nc/4.0/

(c) Article author(s) (or their employer(s) unless otherwise stated in the text of the article) 2018. All rights reserved. No commercial use is permitted unless otherwise expressly granted.

\section{REFERENCES}

1. Taraborelli M, Cavazzana I, Martinazzi N, et al. Organ damage accrual and distribution in systemic lupus erythematosus patients followedup for more than 10 years. Lupus 2017;26:1197-204.

2. Legge A, Doucette S, Hanly JG. Predictors of organ damage progression and effect on health-related quality of life in systemic lupus erythematosus. J Rheumatol 2016;43:1050-6.

3. Bruce IN, O'Keeffe AG, Farewell V, et al. Factors associated with damage accrual in patients with systemic lupus erythematosus: results from the Systemic Lupus International Collaborating Clinics (SLICC) inception cohort. Ann Rheum Dis 2015;74:1706-13.

4. Petri M, Purvey S, Fang $\mathrm{H}$, et al. Predictors of organ damage in systemic lupus erythematosus: the Hopkins Lupus Cohort. Arthritis Rheum 2012;64:4021-8.

5. van Vollenhoven RF, Mosca M, Bertsias G, et al. Treat-to-target in systemic lupus erythematosus: recommendations from an international task force. Ann Rheum Dis 2014;73:958-67.

6. van Vollenhoven R, Voskuyl A, Bertsias G, et al. A framework for remission in SLE: consensus findings from a large international task force on definitions of remission in SLE (DORIS). Ann Rheum Dis 2017;76:554-61.

7. Zen M, Bassi N, Nalotto L, et al. Disease activity patterns in a monocentric cohort of SLE patients: a seven-year follow-up study. Clin Exp Rheumatol 2012;30:856-63.

8. Franklyn K, Lau CS, Navarra SV, et al. Definition and initial validation of a Lupus Low Disease Activity State (LLDAS). Ann Rheum Dis 2016;75:1615-21.

9. Polachek A, Gladman DD, Su J, et al. Defining low disease activity in systemic lupus erythematosus. Arthritis Care Res 2017;69:997-1003. 
10. Golder V, Kandane-Rathnayake R, Hoi AY, et al. Frequency and predictors of the lupus low disease activity state in a multi-national and multi-ethnic cohort. Arthritis Res Ther 2016;18:260.

11. Thamer M, Hernán MA, Zhang Y, et al. Prednisone, lupus activity, and permanent organ damage. J Rheumatol 2009;36:560-4.

12. van Staa TP, Leufkens HG, Cooper C. The epidemiology of corticosteroid-induced osteoporosis: a meta-analysis. Osteoporos Int 2002;13:77787.

13. Mazzantini M, Talarico R, Doveri M, et al. Incident comorbidity among patients with rheumatoid arthritis treated or not with low-dose glucocorticoids: a retrospective study. J Rheumatol 2010;37:2232-6.

14. Ruiz-Arruza I, Ugarte A, Cabezas-Rodriguez I, et al. Glucocorticoids and irreversible damage in patients with systemic lupus erythematosus. Rheumatology 2014;53:1470-6.

15. Apostolopoulos D, Kandane-Rathnayake R, Raghunath S, et al. Independent association of glucocorticoids with damage accrual in SLE. Lupus Sci Med 2016;3:e000157.

16. Gurwitz JH, Bohn RL, Glynn RJ, et al. Glucocorticoids and the risk for initiation of hypoglycemic therapy. Arch Intern Med 1994;154:97.

17. Urowitz MB, Feletar M, Bruce IN, et al. Prolonged remission in systemic lupus erythematosus. J Rheumatol 2005;32:1467-72.

18. Steiman AJ, Urowitz MB, Ibañez D, et al. Prolonged clinical remission in patients with systemic lupus erythematosus. J Rheumatol 2014;41:1808-16.

19. Zen M, laccarino L, Gatto M, et al. Prolonged remission in Caucasian patients with SLE: prevalence and outcomes. Ann Rheum Dis 2015;74:2117-22.
20. Conti F, Ceccarelli F, Perricone C, et al. Flare, persistently active disease, and serologically active clinically quiescent disease in systemic lupus erythematosus: a 2-year follow-up study. PLoS One 2012;7:e45934

21. Drenkard C, Villa AR, Garcia-Padilla C, et al. Remission of systematic lupus erythematosus. Medicine 1996;75:88-98.

22. Swaak AJ, van den Brink HG, Smeenk RJ, et al. Systemic lupus erythematosus: clinical features in patients with a disease duration of over 10 years, first evaluation. Rheumatology 1999;38:953-8.

23. Formiga F, Moga I, Pac M, et al. High disease activity at baseline does not prevent a remission in patients with systemic lupus erythematosus. Rheumatology 1999;38:724-7.

24. Wilhelm TR, Magder LS, Petri M. Remission in systemic lupus erythematosus: durable remission is rare. Ann Rheum Dis 2017;76:547-53.

25. Tsang-A-Sjoe MW, Bultink IE, Heslinga M, et al. Both prolonged remission and lupus low disease activity state are associated with reduced damage accrual in systemic lupus erythematosus. Rheumatology 2017;56:121-8.

26. Nossent J, Kiss E, Rozman B, et al. Disease activity and damage accrual during the early disease course in a multinational inception cohort of patients with systemic lupus erythematosus. Lupus 2010;19:949-56.

27. Zen M, laccarino L, Gatto $M$, et al. The effect of different durations of remission on damage accrual: results from a prospective monocentric cohort of caucasian patients. Ann Rheum Dis 2017;76:562-5 